Research Paper

\title{
MicroRNA-21-5p mediates TGF- $\beta$-regulated fibrogenic activation of spinal fibroblasts and the formation of fibrotic scars after spinal cord injury
}

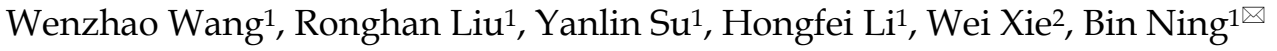 \\ 1. Jinan Central Hospital Affiliated to Shandong University, No. 105, Jiefang Road, Jinan, Shandong, 250013, China; \\ 2. Affiliated Hospital of Taishan Medical University, No. 706, Taishan Street, Taian, Shandong, 271000, China. \\ $\triangle$ Corresponding author: Jinan Central Hospital Affiliated to Shandong University, No. 105, Jiefang Road, Jinan, Shandong, 250013, China. Email: \\ ningbin@sdu.edu.cn, Tel: +86 0531-85695219 \\ (C) Ivyspring International Publisher. This is an open access article distributed under the terms of the Creative Commons Attribution (CC BY-NC) license \\ (https://creativecommons.org/licenses/by-nc/4.0/). See http://ivyspring.com/terms for full terms and conditions.
}

Received: 2017.11.29; Accepted: 2017.12.27; Published: 2018.01.15

\begin{abstract}
Little regeneration of transected axons occurs after the damage caused by traumatic spinal cord injury $(\mathrm{SCl})$, and unidirectional and irreversible fibrotic scars are thought to be the main chemical and physical obstacle for axonal regrowth in $\mathrm{SCl}$ pathology. We previously demonstrated that microRNA (miR)-21-5p and transforming growth factor (TGF)- $\beta 1$, a central pathological mediator of fibrotic diseases, were significantly up-regulated in the lesion epicenter after $\mathrm{SCl}$. Here, we found that TGF- $\beta 1$ enhanced miR-21-5p expression in primary spinal fibroblasts, and regulated the expression of fibrosis-related genes. The overexpression of miR-21-5p promoted the pro-fibrogenic activity of TGF- $\beta 1$ in spinal fibroblasts, while miR-21-5p knockdown attenuated this activity. We identified Smad7 as a target gene of miR-21-5p, suggesting a potential mechanism for the role of miR-21-5p in spinal fibrosis through regulating Smad7 expression. Furthermore, miR-21-5p knockdown in a mouse model significantly improved motor functional recovery after spinal cord injury. These data demonstrate that miR-21-5p functions in an amplifying circuit to enhance TGF- $\beta$ signaling events in the activation of spinal fibroblasts and suggest that miR-21-5p is a potential therapeutic target in the treatment of fibrotic scar formation after SCl.
\end{abstract}

Key words: spinal cord injury, microRNA-21, signaling pathway, fibrotic scar

\section{Introduction}

Traumatic spinal cord injury (SCI) is associated with a significantly high disability and mortality rate, with loss of sensory, motor, and autonomic functions[1]. Currently, there is no effective treatment to promote axon regeneration and restore lost neurologic functions after SCI[2].

Scar tissue is categorized into glial scars and fibrotic scars, and it is thought to be responsible for the failure of transected axons to regenerate in lesions following damage caused by traumatic SCI[3]. The fibrotic component of scar tissue, characterized by the excess deposition of extracellular matrix (ECM) molecules, is non-permissive for axon regeneration[4]. After SCI, adjacent meningeal and perivascular fibroblasts detach and chronically invade the lesion site to form the fibrotic scar. This not only presents as a physical barrier to axon regrowth, but also secretes various inhibitory molecules including tenascin- $C$, ephrinB2, and NG2[3, 5]. Although the role of TGF- $\beta 1$ in scar formation is well documented, the cellular and molecular mechanisms of fibrotic scar formation remain unclear[6].

The TGF- $\beta$ family consists of evolutionarily conserved polypeptides which have important functions in development, tissue homeostasis, and the pathogenesis of diseases, including tumor formation, neuronal degeneration, and scar deposition[6, 7]. TGF- $\beta 1$ is a fibrogenic factor that regulates fibroblast proliferation, differentiation, and apoptosis, and enhances the proliferation and ECM production of 
fibroblasts $[3,8]$. In the TGF- $\beta$ signaling pathway, binding of TGF- $\beta 1$ to the TGF- $\beta$ type II receptor activates the TGF- $\beta$ type I receptor, which phosphorylates the cellular substrates Smad2/3[9]. Phosphorylated Smad2/3 then binds Smad 4 to form a multiprotein complex that translocates into the nucleus and regulates cytokine expression[9, 10]. Smad7 is an inhibitive downstream Smad protein that negatively regulates TGF- $\beta$ signaling, thus rapidly interfering with the activation of $\operatorname{Smad} 2 / 3$ by preventing its connection with the TGF- $\beta$ type I receptor[11, 12].

MicroRNAs (miRNAs) are short (18-22 nucleotides), endogenous, evolutionarily conserved, non-coding RNA molecules that post-transcriptionally regulate gene expression[13]. Primary miRNAs (pri-miRNAs), which can be thousands of base pairs in length, are transcribed from genomic DNA with the aid of RNA polymerase II. Pri-miRNAs are then translated into precursor miRNAs (pre-miRNAs) through the recognition and cleavage function of ribonuclease III[13, 14]. With the assistance of exportin-5, pre-miRNAs are transported from the nucleus to the cytoplasm, forming mature miRNAs. These play critical roles in numerous cytological processes including proliferation, migration, differentiation, and apoptosis[14]. Mature miRNAs regulate target mRNAs by binding to complementary sequences in their $3^{\prime}$ untranslated regions, resulting in target gene silencing through mRNA degradation and/or translational repression[7]. miRNAs have been verified to affect the expression of at least $30 \%-50 \%$ of all protein-coding genes[7]. Individual miRNAs can target and bind to numerous mRNAs, and a given mRNA can be bound by different miRNAs[14, 15]. miRNAs are widely present in a range of tissues and fluids such as muscle, the skeleton, gray matter, plasma, serum, urine, and cerebrospinal fluid[16, 17]. They also play a critical role in regulating tissue fibrosis, and are involved in matrix repair, the secretion of collagens, the phosphorylation of Smad signaling proteins[7], and neurological and neurodegenerative diseases.

miR-21 plays a major role in tissue pathological and physiological processes, and is an important regulator of molecular networks[18]. Recent studies have revealed a role for miR-21 in the fibrosis of various tissues, including cardiac, lung, liver, kidney, tumor, and skin[19]. Moreover, miR-21 was shown to be upregulated following several types of central nervous system (CNS) injuries including traumatic CNS injury $[17,20]$. Pathological roles for miR-21 have also been identified in a murine model of SCI, including regulation of the non-cell autonomous pathway of astrocytes and the regrowth of axons in glial scars.[21]

The present study used a mouse model of spinal cord contusion injury in which primary spinal meninges fibroblasts were separated and purified from the lesions to verify our hypothesis that miR-21-5p regulates the formation of fibrotic scars and axonal regeneration after SCI through the TGF- $\beta$ signaling pathway by targeting Smad7. We used a $\mu$ ParaFlo microRNA microarray assay and quantitative real-time (qRT)-PCR to show that miR-21-5p is significantly up-regulated in the lesion tissues of a mouse SCI model. Kyoto Encyclopedia of Genes and Genomes (KEGG) pathway analysis indicated that miR-21-5p specifically binds to Smad7, while bioinformatics analysis based on TargetScan, PicTar, and miRanda miRNA databases revealed a Smad7 targeting sequence (AUAAGCUA) in miR-21-5p. miR-21-5p was shown to negatively regulate Smad7, achieving pro-fibrogenic effects. These findings suggest that miR-21-5p is a promising target for regulating the fibrosis of spinal meninges fibroblasts, and may provide an alternative treatment for SCI.

\section{Materials and Methods}

\section{Cell culture and transfection}

Primary mouse spinal fibroblasts (PriCells, Wuhan, China) were cultured at $37^{\circ} \mathrm{C}$ in a humidified atmosphere of $5 \% \mathrm{CO}_{2}$ in modified Eagle's medium (Gibco, Shanghai, China) supplemented with $8 \%$ fetal bovine serum (Gibco, Brisbane, Australia) and 100 $\mathrm{IU} / \mathrm{mL}$ penicillin-streptomycin (Solarbio, Beijing, China). Cells from passages 2-4 were used for the experiments. After culturing to $60 \%-80 \%$ confluency, the cells were treated with different concentrations of TGF- $\beta(0,1.25,2.5,5$, and $10 \mathrm{ng} / \mathrm{ml})$ for various time courses $(0,6,12,24$, and $48 \mathrm{~h})$. Transfection of miR-21-5p mimics/ inhibitors/ scrambled controls (RiboBio, Guangzhou, China) was initiated when the cells were $30 \%-50 \%$ confluent. After $48 \mathrm{~h}$, the cells were stimulated with TGF- $\beta(10 \mathrm{ng} / \mathrm{ml})$ for $48 \mathrm{~h}$.

\section{Mouse model and functional assessment}

All animal procedures were approved by the Research Ethics Committee of Shandong University (Jinan, China). Adult male C57BL/ 6 mice (8-10 weeks old) were purchased from the Laboratory Animal Centre of Shandong University. The animals were housed in a clean facility under the controlled conditions of $22-24^{\circ} \mathrm{C}$, relative humidity $30 \%-50 \%$, and a 12-h light-dark cycle. A standard mouse diet and filtered water were available ad libitum.

Mice were anesthetized with 3\% pentobarbital (30 mg/ kg), then a T8-T10 vertebral laminectomy was performed to expose the spinal cord. Moderate SCI 
was performed using a modified Allen's weight drop apparatus ( $8 \mathrm{~g}$ weight at $50 \mathrm{~mm}, 8 \mathrm{~g} \times 50 \mathrm{~mm}$ ). For the sham group, the same laminectomy was performed but the mice did not receive a moderate contusion injury. The success of the compression was confirmed by tail spasms, retraction-like flutters of the legs, and delayed paralysis. After laminectomy, mice were kept on a heated pad for $24 \mathrm{~h}$, and bladders were manually voided twice daily.

A total of 84 mice were used in vivo assays. First batch of 24 mice were divided into two groups: those in the sham group $(\mathrm{n}=12)$ underwent laminectomy without spinal cord contusion; those in the SCI group $(\mathrm{n}=12)$ underwent spinal cord contusion without further treatment. After 3 days, mice were killed by excess pentobarbital, and $\sim 0.5 \mathrm{~cm}$ of the spinal cord containing vertebrae T8-T10 was removed. These mice were used for microarray assay, qRT-PCR, and immunohistochemistry. The second bunch of mice (n $=12$ ) with spinal cord contusion were used for hematoxylin and eosin (H\&E) stain. Spinal cord tissues were harvested at the lesion epicenter of the injury on days $1,3,7$, or 14 post-surgery. An additional 48 mice were used for oligonucleotide transfection assay and antagomir-21 and antagomir-21-negative control (miR-21 NC) were used for intrathecal injection. Antagomirs can effectively and lastingly silence endogenous miRNAs in vivo[22]. Mice were randomly divided into the following four groups: those in the sham group ( $\mathrm{n}=$ 12) underwent laminectomy without SCI; those in the SCI group $(n=12)$ underwent spinal cord contusion; those in the miR-21 NC group $(n=12)$ underwent spinal cord contusion and were treated intrathecally with miR-21 NC ( $50 \mu \mathrm{L} / \mathrm{d}, 100 \mathrm{nmol} / \mathrm{mL}$; RiboBio) for 3 days; and those in the antagomir-21 group $(n=12)$ were given a intrathecal injection of antagomir-21 (50 $\mu \mathrm{L} / \mathrm{d}, 100 \mathrm{nmol} / \mathrm{mL}$; RiboBio) for 3 days post-spinal cord contusion. Locomotor activity was evaluated in an open field for 14 days using the Basso Mouse Scale (BMS), which is a 9-point scale designed to assess the recovery of hindlimb locomotor function after SCI in mice. Trunk position and stability, paw placement, toe clearance, stepping coordination, hindlimb joint movements, and tail position were assayed by four independent experienced investigators who were blinded to the treatment conditions. They observed movement and scored locomotor function according to the BMS standard. Mice were sacrificed by an overdose injection of 3\% pentobarbital (i.p.). Spinal cord tissues were harvested at the lesion epicenter of the contusion injury on days 14 post-surgery.

\section{RNA isolation and qRT-PCR}

Total RNA was isolated from tissues and fibroblasts using RNAiso ${ }^{\mathrm{TM}}$ Plus (Takara, Dalian, China). The Bulge-Loop ${ }^{\mathrm{TM}}$ miRNA reverse transcription kit (RiboBio), the Bulge-Loop ${ }^{\mathrm{TM}}$ miRNA qRT-PCR Starter Kit (RiboBio), and miR-21-5p primers (RiboBio) were used to measure the expression levels of miR-21-5p; U6 snRNA (RiboBio) was used as an endogenous control to quantify and normalize the results. The Takara PrimeScript RT Reagent kit (Takara) was used for reverse transcription. The expression of Smad7, type I a1 collagen (Col1a1), type I a2 collagen (Col1a2), fibronectin $(F N)$, brain-derived neurotrophic factor (BDNF), and neurotrophin (NGF) was measured by qRT-PCR with SYBR Premix Ex Taq ${ }^{\mathrm{TM}}$ (Takara) using the 7500 real-time PCR system analyzer (Applied Biosystems; Thermo Fisher Scientific, Wilmington, DE). The expression of GAPDH was used as the internal control to normalize the data. The value 2 $-\triangle \triangle C T$ was used for comparative quantitation. All PCR reactions were performed in triplicate. The following primer pairs were used:

Mouse Smad7 forward: 5'-CTGCTGTGCAAAG TGTTCAGG-3', reverse: 5'-CCATTGGGTATCTGGA GTAAGGA-3';

Mouse Col1a1 forward:5'-GACATGTTCAGCT TTGTGGACCTC-3', reverse: 5'-GGGACCCTTAGGC CATTGTGTA-3';

Mouse Col1a2 forward: 5'-TGCTTGCAGTAA CTTCGTGCCTA-3', reverse: 5'-CATGGGACCATC AACACCATC-3';

Mouse fibronectin forward: 5'-GCTTTGGCAGT GGTCATTTCAG-3', reverse: 5'-ATTCCCGAGGCAT GTGCAG-3';

Mouse BDNF forward 5'-TCAAGTTGGAAGC CTGAATGAATG-3', reverse: 5'-CTGATGCTCAGG AACCCAGGA-3';

Mouse NGF forward: 5'-TGCCAAGGACG CAGCTTTC-3', reverse 5'-TGAAGTTTAGTCC AGTGGGCTTCAG-3';

Mouse GAPDH forward: 5'-TGTGTCCGTC GTGGATCTGA-3', reverse: 5'-TTGCTGTTGAAGT CGCAGGAG-3'.

\section{Microarray assay and bioinformatics analysis}

Total RNA was isolated from spinal cords harvested at day 3 after SCI. Microarray assays were performed by a service provider (LC Sciences Co., Hangzhou, China).

TargetScan (http:/ / www.targetscan.org), PicTar (http:/ / pictar.mdc-berlin.de/), and miRanda (http:/ / microrna.sanger.ac.uk) bioinformatics indicated the potential seed sequences for miRNA. KEGG Pathway analysis (www.genome.jp/kegg) indicated potential miRNA networks and target genes $(P<0.05)$. 


\section{Protein isolation and western blotting}

Fibroblasts and spinal cord tissues were homogenized on ice in $200 \mu \mathrm{l}$ of Cell Lysate buffer (Solarbio) containing $1 \mathrm{mM}$ phenylmethane sulfonyl fluoride and protein phosphatase inhibitor solution (1×) (Solarbio). All crude sample buffers were centrifuged at $18,000 \times g$ at $4^{\circ} \mathrm{C}$ for $15 \mathrm{~min}$, then the supernatant samples containing $1 \mathrm{mg}$ protein/500 $\mu \mathrm{l}$ were subjected to sodium dodecylsulfate-polyacrylamide gel electrophoresis (Solarbio) and transferred onto polyvinylidene fluoride membranes (Millipore, Billerica, MA, USA). After being blocked with 2\% bovine serum albumin (Thermo Fisher Scientific), the blots were incubated with anti-fibronectin antibody (1:5,000; Abcam, Cambridge, USA), anti-Smad7 antibody (1:500; Cell Signaling Technology, Danvers, USA), and anti-GAPDH antibody (1:5,000; Abcam) at $4^{\circ} \mathrm{C}$ overnight. After washing, the membranes were probed with goat anti-rabbit horseradish peroxidaseconjugated secondary antibody (1:5,000; Proteintech, Wuhan, China) at room temperature for $45 \mathrm{~min}$ and visualized by West Pico ECL Substrate (Solarbio).

\section{Histology and immunohistochemistry}

Spinal cord samples were fixed overnight in $4 \%$ buffered paraformaldehyde solution. After fixation, tissues were processed routinely by washing, dehydration, clearing, embedding, and freezing at the optimal cutting temperature. Slices $(5 \mu \mathrm{m})$ were sectioned by a vibratome and stained with H\&E. For immunohistochemistry, spinal cord tissue sections were deparaffinized with xylene and hydrated in ethanol solutions before heating in citrate buffer. After three extensive washes with phosphate-buffered saline (PBS), they were heated in citrate buffer for antigen retrieval, and blocked in $5 \%$ goat serum for 45 min. After rinsing in PBS, the sections were incubated overnight at $4^{\circ} \mathrm{C}$ with one of the following primary antibodies: chondroitin sulfate proteoglycans (CSPGs) (1:200; Abcam) or Smad7 (1:500; Cell Signaling Technology). Subsequently, sections were incubated in horseradish peroxidase-labeled goat anti-rabbit IgG secondary antibody (1:2,500; Proteintech) for $20 \mathrm{~min}$ at $37^{\circ} \mathrm{C}$. After three extensive washes with PBS, the sections were stained with 3,3'-diaminobenzidine tetrahydrochloride (Gene Tech, Shanghai, China), and analyzed under an Olympus microscope (Olympus Corporation, Tokyo, Japan).

\section{Statistical analysis}

GraphPad Prism software (GraphPad Software, La Jolla, CA, USA) and SPSS (IBM, Chicago, IL, USA) were used for statistical analysis. Data were expressed as means \pm standard error of the mean. One-way analysis of variance with Dunnett's post hoc test was used for multiple comparisons. Statistical significance was determined at $P<0.05$.

\section{Results}

\section{miR-21-5p and TGF- $\beta$ expression after $\mathrm{SCl}$ in vivo}

For the preliminary determination of miRNA expression differences between the sham group and SCI group, we used $\mu$ ParaFlo microarray analysis. A total of 157 differentially expressed miRNAs were identified, of which 96were upregulated and 61were downregulated in the SCI group compared with the sham group. All differentially expressed miRNAs showed a $>2.0$-fold-change threshold and $P<0.05$ (Figure 1A). Most strikingly, the expression of miR-21-5p in SCI tissues was significantly higher than in the sham group; this was also confirmed by qRT-PCR (Figure 1B). Immunohistochemistry detected substantially increased staining of TGF- $\beta 1$ after SCI (Figure 1D). Based on our KEGG data, we drew the diagram of an miR-21-5p gene regulatory network based on target genes in TGF- $\beta$ signaling pathway, 21 putative target genes of miR-21-5p were involved and Smad7 could play a key role in activation of the TGF- $\beta$ signaling pathway (Figure 1C). Bioinformatics analysis using TargetScan, PicTar, and miRanda demonstrated that Smad7 contained a targeting sequence (AUAAGCUA) for miR-21-5p.

\section{TGF- $\beta$ regulated the expression of $m i R-21-5 p$ and fibrosis-related genes}

TGF- $\beta 1$ expression has been shown to be an important mediator of fibrosis and the active synthesis of ECM proteins[23, 24]. Experiments on the dose curve and time course were performed to optimize the appropriate TGF- $\beta 1$ concentration and treatment time for primary spinal cord fibroblast activation. Fibroblasts were treated with TGF- $\beta 1$ at the indicated concentration $(0,1.25,2.5,5$, and 10 $\mathrm{ng} / \mathrm{ml}$ ) for $48 \mathrm{~h}$. We previously observed that a dose of TGF- $\beta 1$ exceeding $10 \mathrm{ng} / \mathrm{ml}$ may cause adverse effects on the proliferation of primary spinal fibroblasts (unpublished observations). The expression of fibrosis-related genes COL1a1, COL1a2, and $F N$ was increased dose-dependently, with the highest expression seen at $10 \mathrm{ng} / \mathrm{ml}$ TGF- $\beta 1$ (Figure $2 \mathrm{~A}-\mathrm{C})$. The optimal duration of TGF- $\beta 1$-induced fibroblast activation was found to be $48 \mathrm{~h}$ (Figure 2D-F). miR-21-5p expression was detected by qRT-PCR after exposure to TGF- $\beta 1$. It increased $48 \mathrm{~h}$ after TGF- $\beta 1$ treatment, which correlated with the decreased expression of Smad7 (Figure 2I-L). FN protein expression was also increased in a dose- and time-dependent manner, but Smad7 expression was 
decreased $48 \mathrm{~h}$ after TGF- $\beta 1$ treatment (Figure 2G, H,

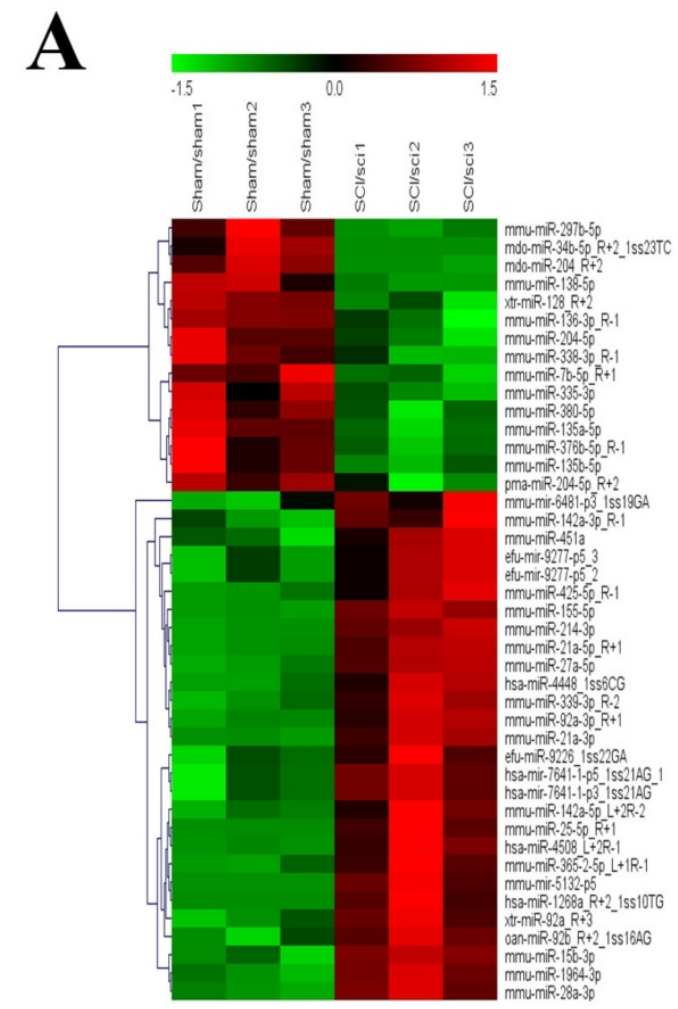

M).

B

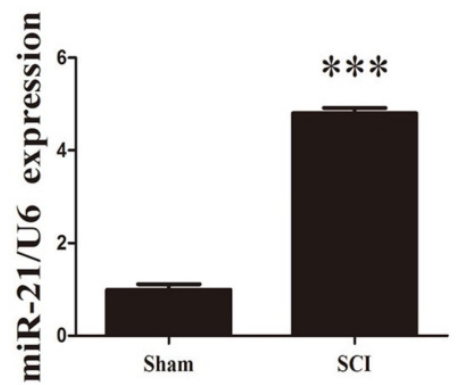

C

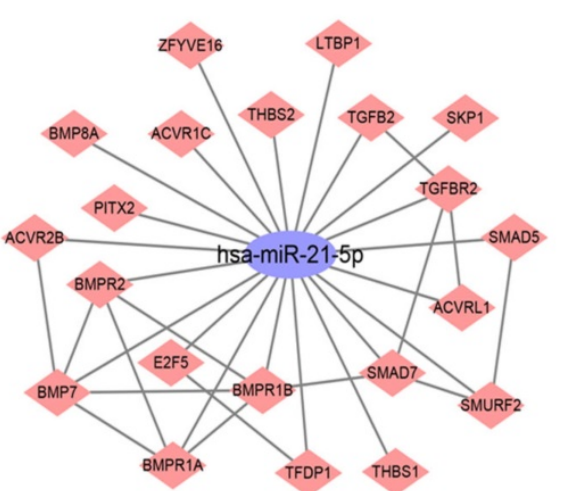

D

TGF- $\beta$
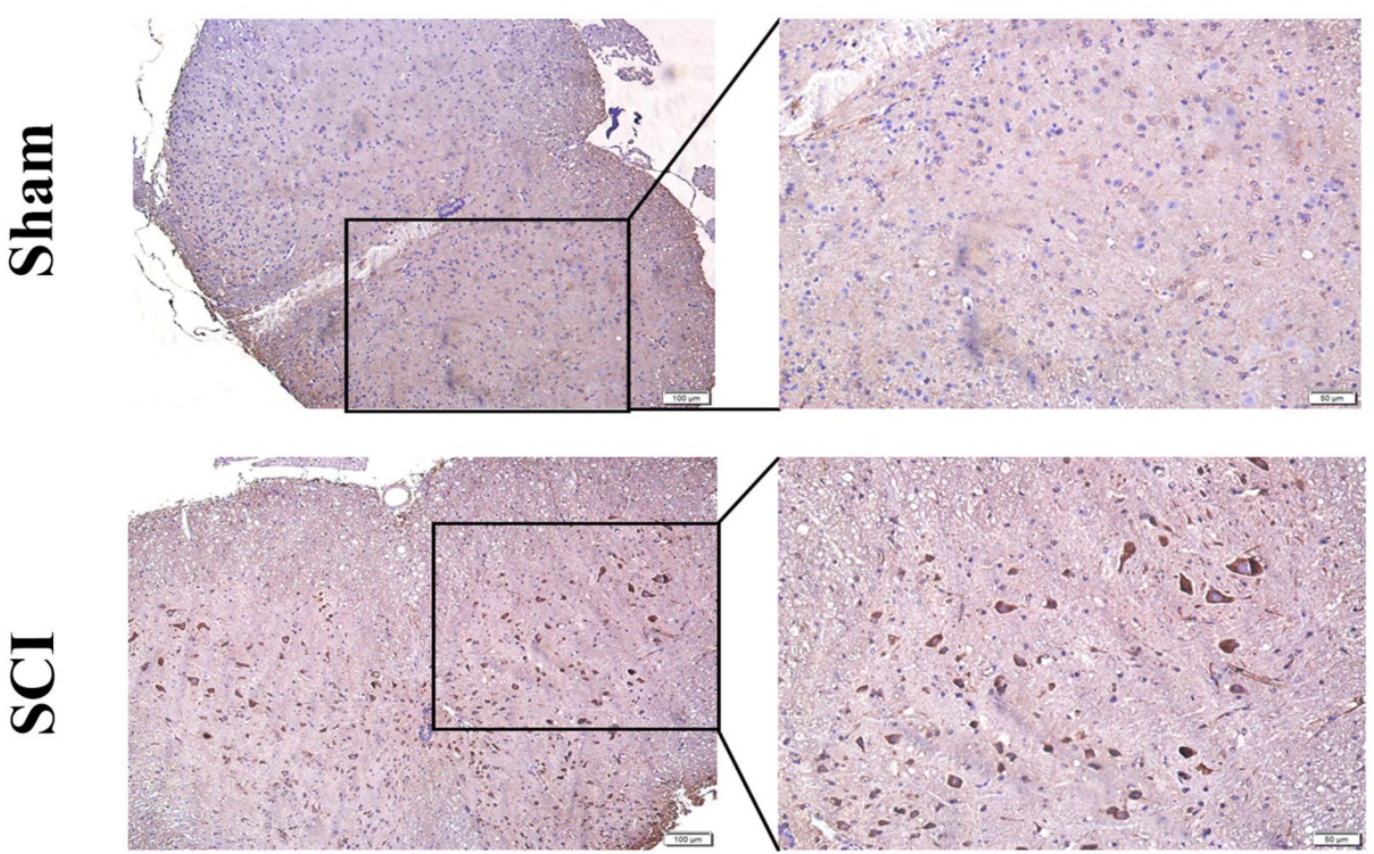

Figure 1. miR-21-5p and TGF- $\beta 1$ were up-regulated in the lesion epicenter after SCI. (a) Heat map showing the expression levels of differentially expressed miRNAs in the lesion epicenter 3 days after $\mathrm{SCl}$. Genes are shown in the right column. Red pixels correspond to an increased abundance of the gene in the indicated sample, whereas green pixels indicate decreased levels; fold-change $>2.0$ and $P<0.05$. (b) miR-21-5p expression was validated by qRT-PCR. Data are shown as mean \pm SEM, $* * * P<0.001$. (c) The miR-21-5p gene network (TGF- $\beta$ signaling pathway) was determined based on KEGG pathway analyses. The constructed subnetwork of miR-21-5p and its potential target genes shows an enrichment of genes involved in the TGF- $\beta$ signaling pathway. (d) Immunohistochemical staining with TGF- $\beta 1$ antibodies in the sham group and in samples 3 days after $\mathrm{SCl}$. 
A

D

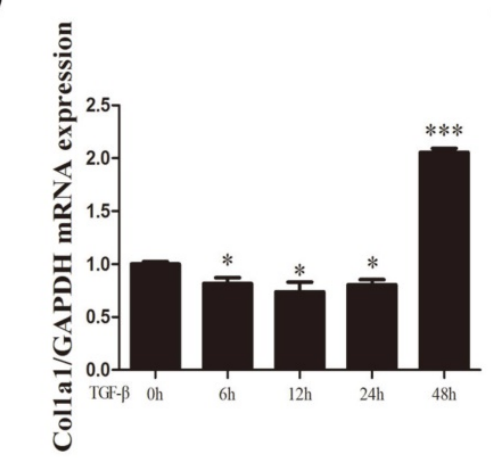

G

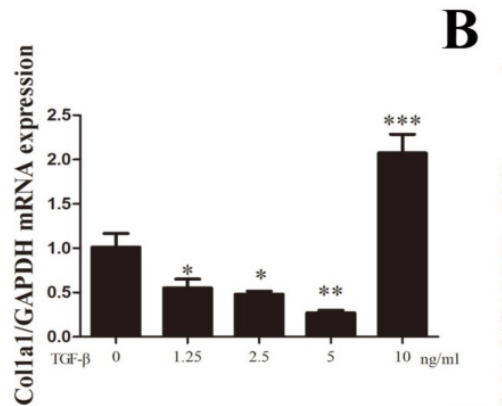

E

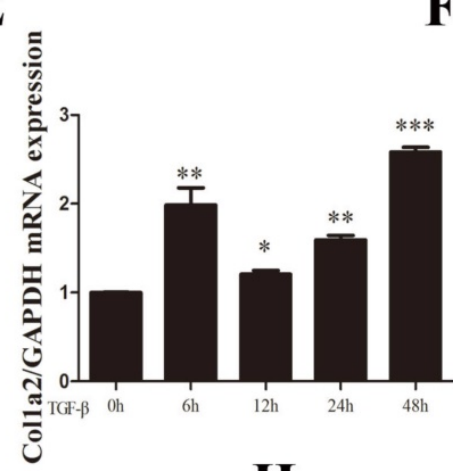

C

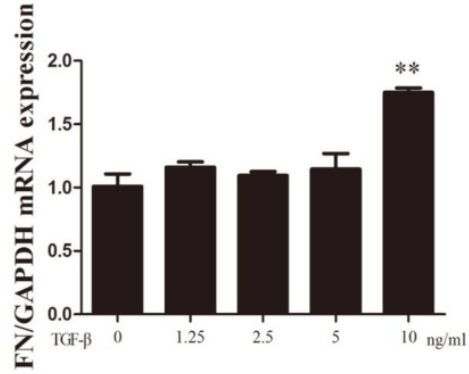

$\mathbf{F}$

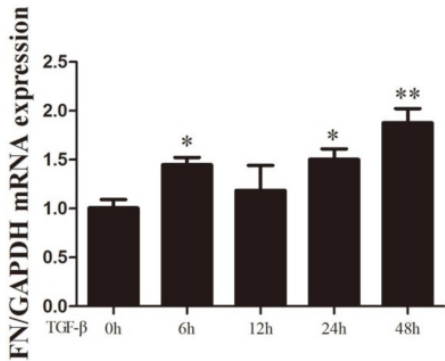

\section{H}

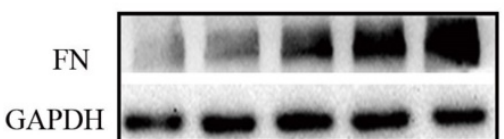

$\begin{array}{llllll}\text { TGF- } \beta & 0 & 1.25 & 2.5 & 5 & 10 \mathrm{ng} / \mathrm{ml}\end{array}$

GAPDH
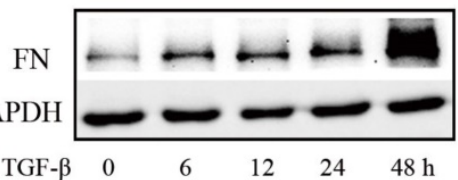

I
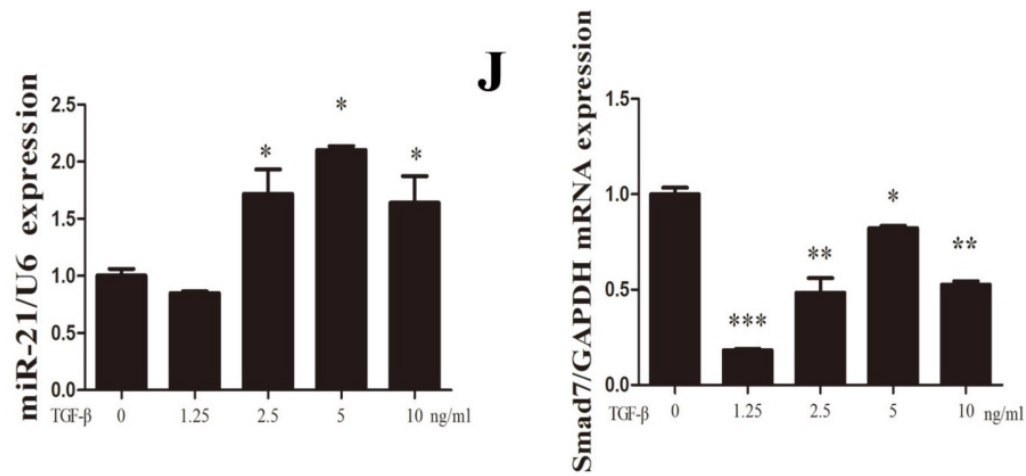

K

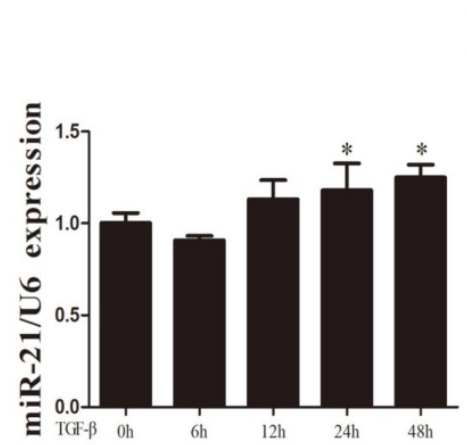

$\mathbf{L}$

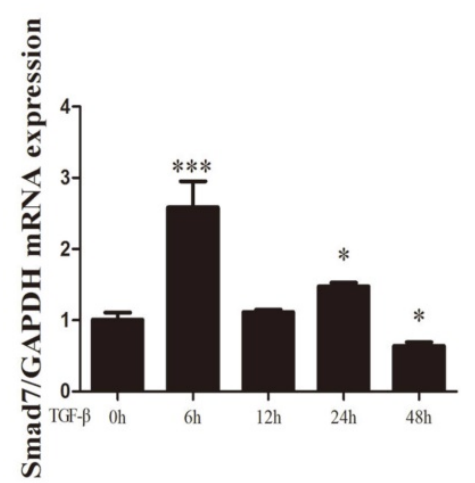

\section{M}

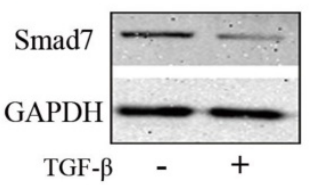

Figure 2. Expression of miR-21-5p and fibrosis-related genes was regulated by TGF- $\beta 1$. Fibroblasts were treated with TGF- $\beta 1$ at the indicated concentration $(0$, $1.25,2.5,5$, and $10 \mathrm{ng} / \mathrm{ml})$ or different time periods $(0,6,12,24$, and $48 \mathrm{~h})$. (a-f) The expression of fibrosis-related genes COLI $\alpha I$, COLI $\alpha 2$, and FN expression was detected by qRT-PCR. $(\mathbf{g}, \mathbf{h})$ Western blot of FN following treatment with different concentrations and time courses of TGF- $\beta$. (i-I) miR-21-5p is induced by TGF- $\beta$ (a and d); the expression pattern of Smad7 is shown. (h) Cells were stimulated with $10 \mathrm{ng} / \mathrm{ml}$ TGF- $\beta$ for $48 \mathrm{~h}$, then protein levels of Smad7 and GAPDH were determined. Data are expressed as the mean \pm SD. $* P<0.05$, $* * P<0.01$, $* * * P<0.001$. 
A

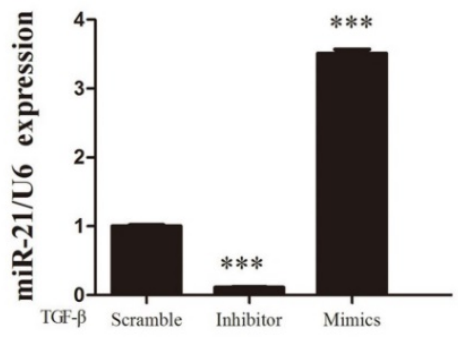

D

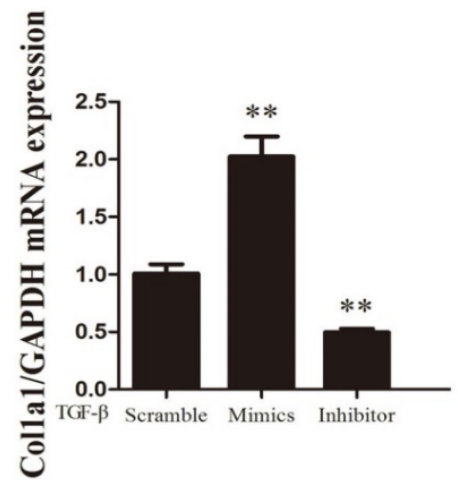

I

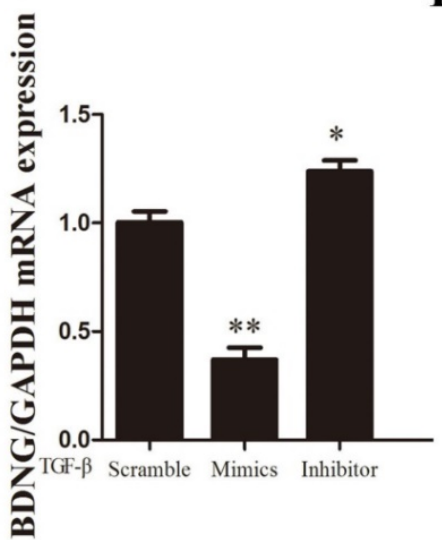

B

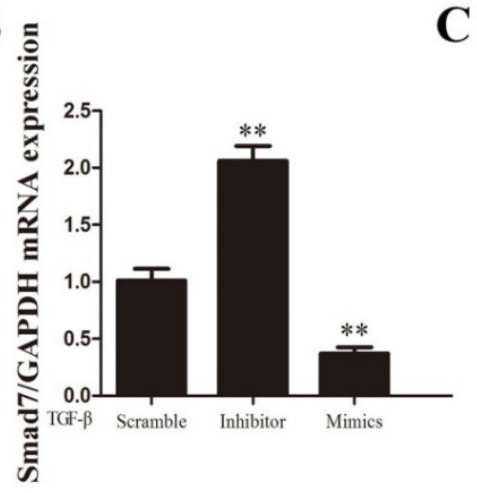

E

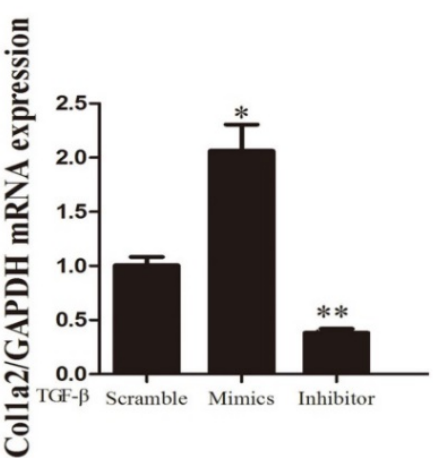

H

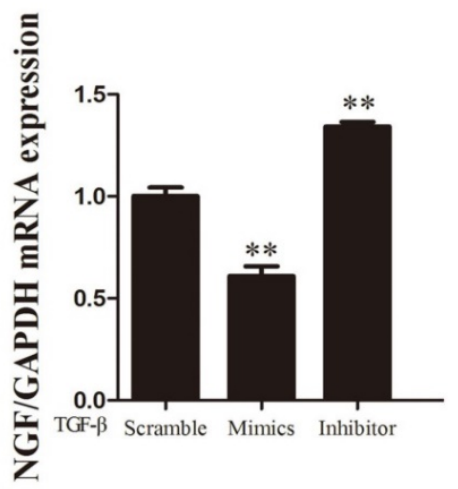

C

F

G
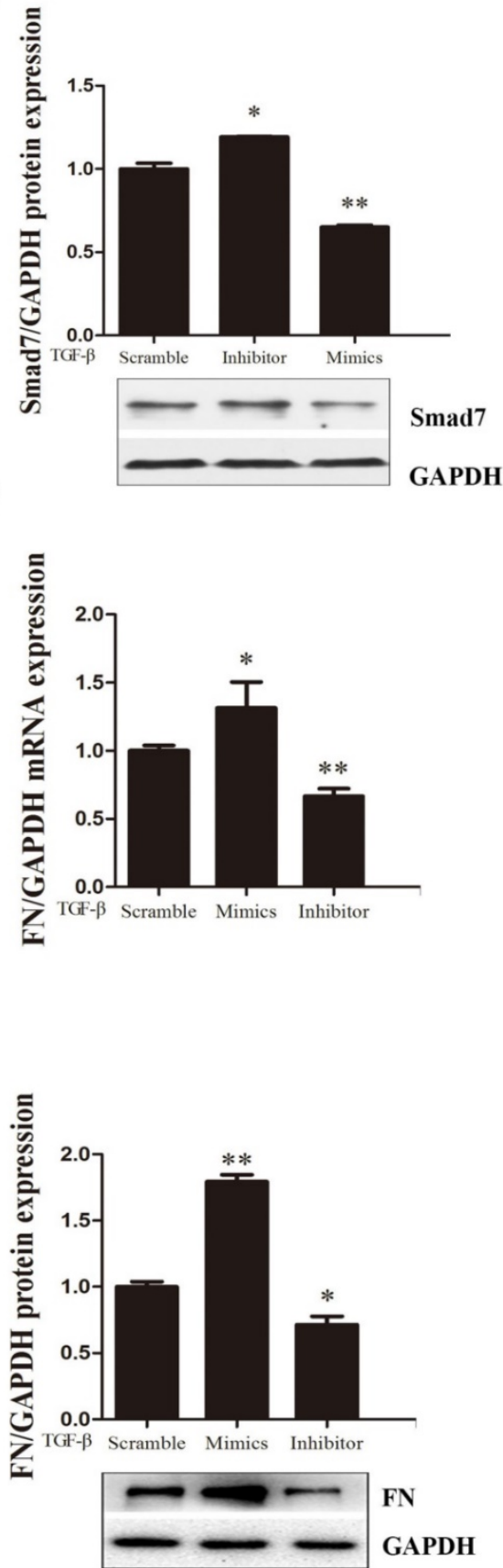

Figure 3. Expression of fibrosis-related genes was regulated by the overexpression or knockdown of miR-21-5p. After treatment with $10 \mathrm{ng} / \mathrm{ml}$ TGF- $\beta$ for $48 \mathrm{~h}$, fibroblasts were transfected with miR-21-5p mimics, miR-21-5pinhibitors, or scrambled controls. (a, b) miR-21-5p levels and mRNA levels of Smad7 were determined by qRT-PCR. (c) Smad7 protein levels were determined by western blotting. (d-f) mRNA expression levels of FN, COLIal, and COLI 2 were determined by qRT-PCR. (g) FN protein levels were determined by western blotting. (h, i) The expression of BDNF and NGF was detected by qRT-PCR. Data are expressed as means \pm SD. $* P<0.05$, $* * P<0.01$, $* * * P<0.001$.

\section{miR-21-5p regulates fibrotic scar-related gene expression in primary spinal fibroblasts}

To investigate the effects of miR-21-5p in TGF- $\beta 1$-induced fibroblast activation, miR-21-5p was knocked down or over-expressed in primary spinal fibroblasts. RT-PCR was used to ensure the successful transduction of miR-21-5p mimics and inhibitor (Figure 3A). The expression of fibrosis-related genes
COL1a1, COL1a2, and FN was detected by qRT-PCR, and protein levels were determined by western blotting. We previously demonstrated that miR-21-5p over-expression induced the expression of fibrosis-related genes following TGF- $\beta 1$ stimulation in primary spinal fibroblasts. Conversely, in this study, qRT-PCR showed that miR-21-5p knockdown suppressed TGF- $\beta 1$-induced fibrosis-related gene expression (Figure 3D-G). 
The effects of miR-21-5p on Smad7 expression were further analyzed by western blotting and qRT-PCR during fibroblast activation. miR-21-5p over-expression significantly decreased the level of Smad7 expression, while miR-21-5p knockdown upregulated TGF- $\beta 1$-induced Smad7 expression (Figure 3B, C). RT-PCR was used to evaluate whether miR-21-5p regulated the expression of neuroprotective factors. BDNF and NGF were shown to be expressed at high levels in miR-21-5p knockdown groups following TGF- $\beta 1$ induction, and were suppressed in over-expressing groups (Figure $3 \mathrm{H}, \mathrm{I})$.

\section{miR-21-5p regulated functional recovery after traumatic SCI}

To further confirm whether miR-21-5p is a critical mediator of neurologic functions after SCI, miR-21-5p knockdown SCI animal experiments were performed. Contusion injuries on the spinal cord of inbred C57 mice were produced using a standard Allen's impactor. H\&E staining demonstrated that the structural integrity of the spinal cord lesion epicenter was severely damaged after SCI, including hemorrhages, inflammatory cell activation, and vacuolar and neuronal degeneration (Figure 4A).Antagomir-21 and the homologous negative control were transfected to the lesion epicenter of C57 mice after SCI, and their successful transduction was determined by the RT-PCR analysis of miR-21-5p expression. Antagomir-21 significantly reduced the expression of miR-21-5p in the lesion area of the spinal cord 3 days post-injection (Figure 4B). To examine the function of miR-21-5p in SCI pathology, the hindlimb motor function was assessed using the BMS from day 1 post-surgery. The motor function of the sham group showed an improvement on day 2 and had returned to normal on day 3 post-surgery. The antagomir-21 group started to show a better improvement in motor function 7 days after $\mathrm{SCI}$, and this became more significant 14 days after SCI compared with the miR-21 NC group. Consequently, we suggest that antagomir-21 has a significant functionality recovery effect after $\mathrm{SCI}$, compared with controls (Figure 4C). Immunohistochemistry on day 14 post-surgery showed that Smad7 and CSPGs were downregulated by the inhibition of miR-21-5p expression (Figure 4D). qRT-PCR showed that miR-21-5p knockdown significantly increased the expression of NGF and BDNF (Figure 4E).

\section{Discussion}

Following traumatic SCI, little regeneration of transected axons occurs. This may reflect the influence of myelin-associated external inhibitory factors, fibrotic scars and astrocytic scars formed at the lesion site[25], a lack of supporting and neurotrophic factors[26], or the absence of mature neural intrinsic growth[27]. It has long been thought that astrocytic scars are a barrier to axon regeneration, particularly because astrocytes secrete CSPGs which are efficient axon regeneration inhibitors[25, 28]. However, recent studies suggest that astrocytic scars are fundamental supporting factors for axon regeneration that have beneficial roles in the subacute phase after SCI in processes such as local immune regulation, neuroprotection, and tissue repair[29-31]. Additionally, substantive amounts of critical CSPGs were shown to be produced by non-astrocyte cells, especially fibroblast and macrophage cells[29], which are the fundamental units of fibrotic scars. After SCI, fibroblasts detach from meninges, and perivascular cells invade and reside in the lesion site. Fibroblasts then multiply and express ECMs including fibronectin, type I collagen, type IV collagen, and laminin to form the fibrotic scar[32]. We propose that the fibrotic scar itself is the main chemical and physical obstacle for axonal regrowth in the lesion epicenter.

Fibrotic scars are thought to be unidirectional and irreversible in SCI pathology, and their formation is suggested to be regulated by complex and combinatorial inter- and intracellular signaling mechanisms. TGF- $\beta 1$, a key cytokine for fibrosis which shows increased expression after SCI, contributes to the activation of spinal fibroblasts in vitro. TGF- $\beta 1$ expression increases around the lesion site during the period of fibrotic scar formation, peaks at 4 days, then declines but remains at enhanced levels 2 weeks after a CNS injury[23]. Increased TGF- $\beta 1$ expression leads to a marked rise in transcriptional cascades downstream of $\mathrm{p}-\mathrm{Smad} 2 / 3$ activation[6, 27], and the enhanced expression of fibrosis-related genes. Interestingly, recent findings indicate that Col I genes are not only the most highly expressed ECM genes in the subacute phase of SCI, but are also involved in astrocyte adhesion and scar formation[33].

In this study, we show that the TGF- $\beta$ signaling pathway is significantly and selectively activated during formation of the fibrotic scar. Following SCI, the expression of miR-21-5p and TGF- $\beta 1$ is upregulated, while that of Smad7 is downregulated. Smad7 is an inhibitory Smad protein that negatively regulates TGF- $\beta$ signaling pathway activation and functions by targeting the TGF- $\beta 1$ receptor[12, 34, 35]. Our bioinformatics analysis and KEGG data showed that putative target genes of miR-21-5p that were underexpressed or overexpressed are involved in several signaling pathways associated with SCI. 
Among these, the TGF- $\beta$ signaling pathway is the

putative target gene of miR-21-5p.

most relevant pathway, and we identified Smad7 as a

A

Sham

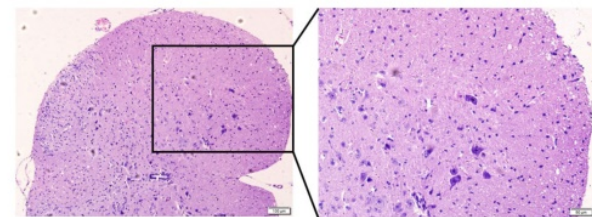

$\mathrm{SCI}$

$7 \mathrm{D}$

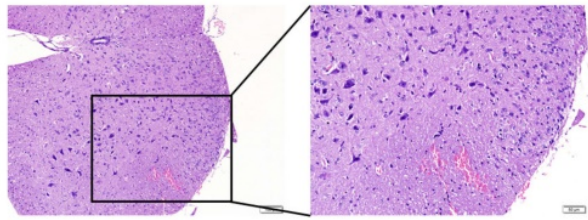

SCI

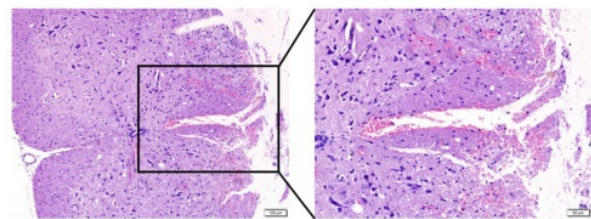

SCI

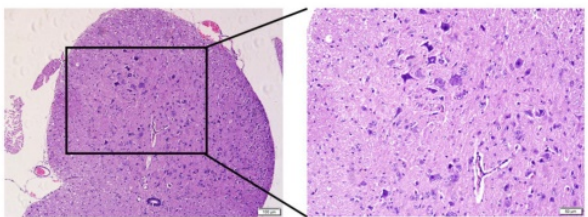

B

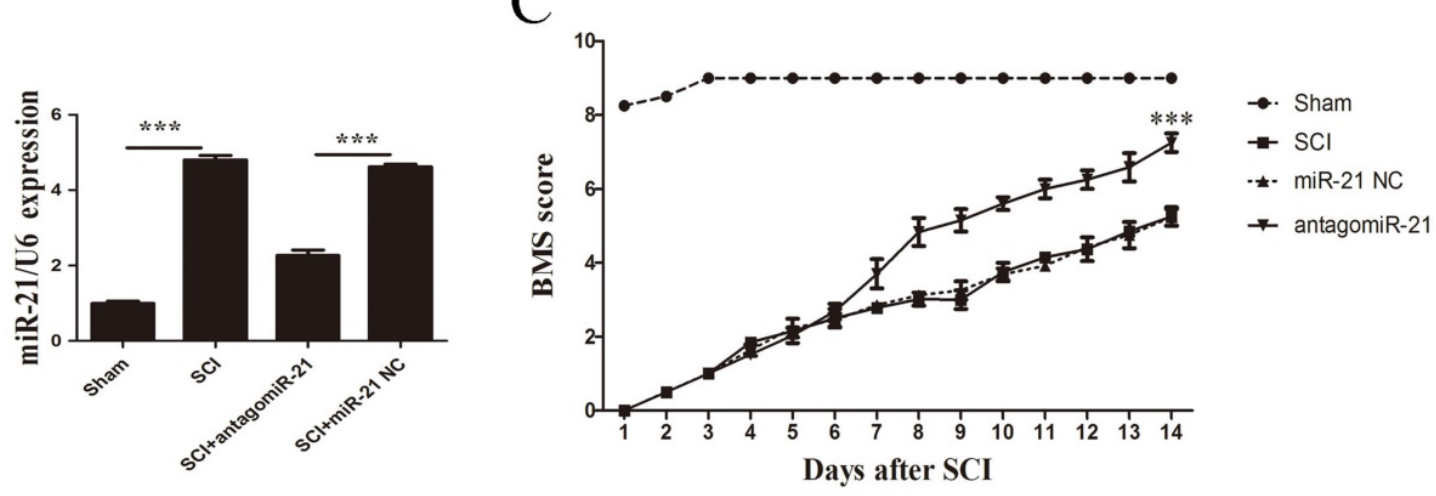

C

$\mathrm{D}$

$\mathrm{SCI}+$

miR-21 NC

Smad7

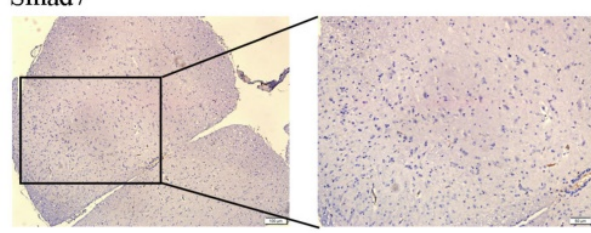

$\mathrm{SCI}+$

antagomiR-21

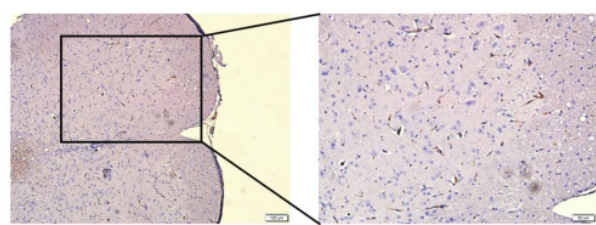

$\mathrm{E}$

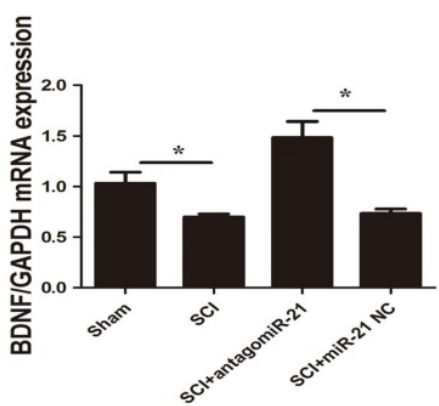

CSPG
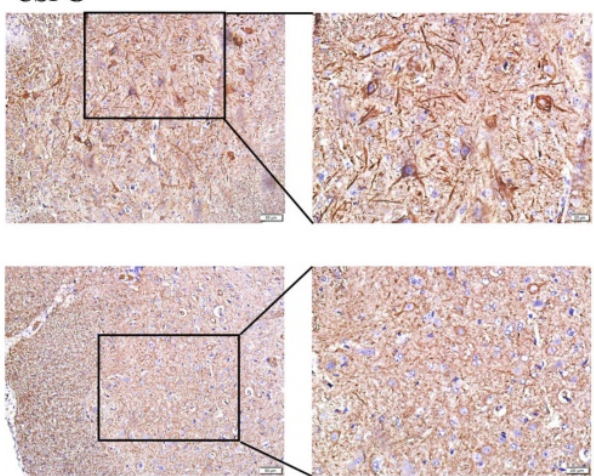

F

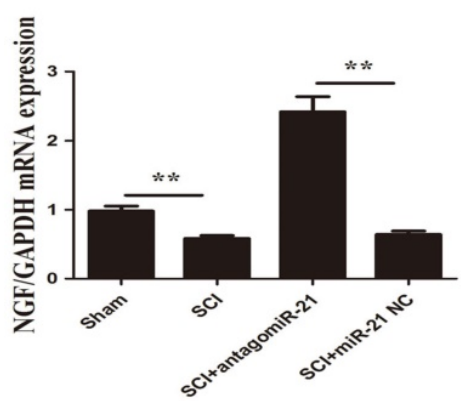

Figure 4. miR-21-5p regulated functional recovery after traumatic spinal cord injury. (a) Hematoxylin and eosin staining of the spinal cord in sham and SCl groups at days 1, 7, and 14 post surgery. (b) miR-21-5p levels were determined by qRT-PCR. (c) BMS scores indicate the motor functional index over 14 days after SCI. Data are shown as mean \pm SEM, ***P<0.001. (d) Immunohistochemistry to determine the expression of Smad7 and CSPGs. (e) BDNF and NGF expression was detected by qRT-PCR. Data are expressed as the mean \pm SD. $* P<0.05$, $* * P<0.01$. 


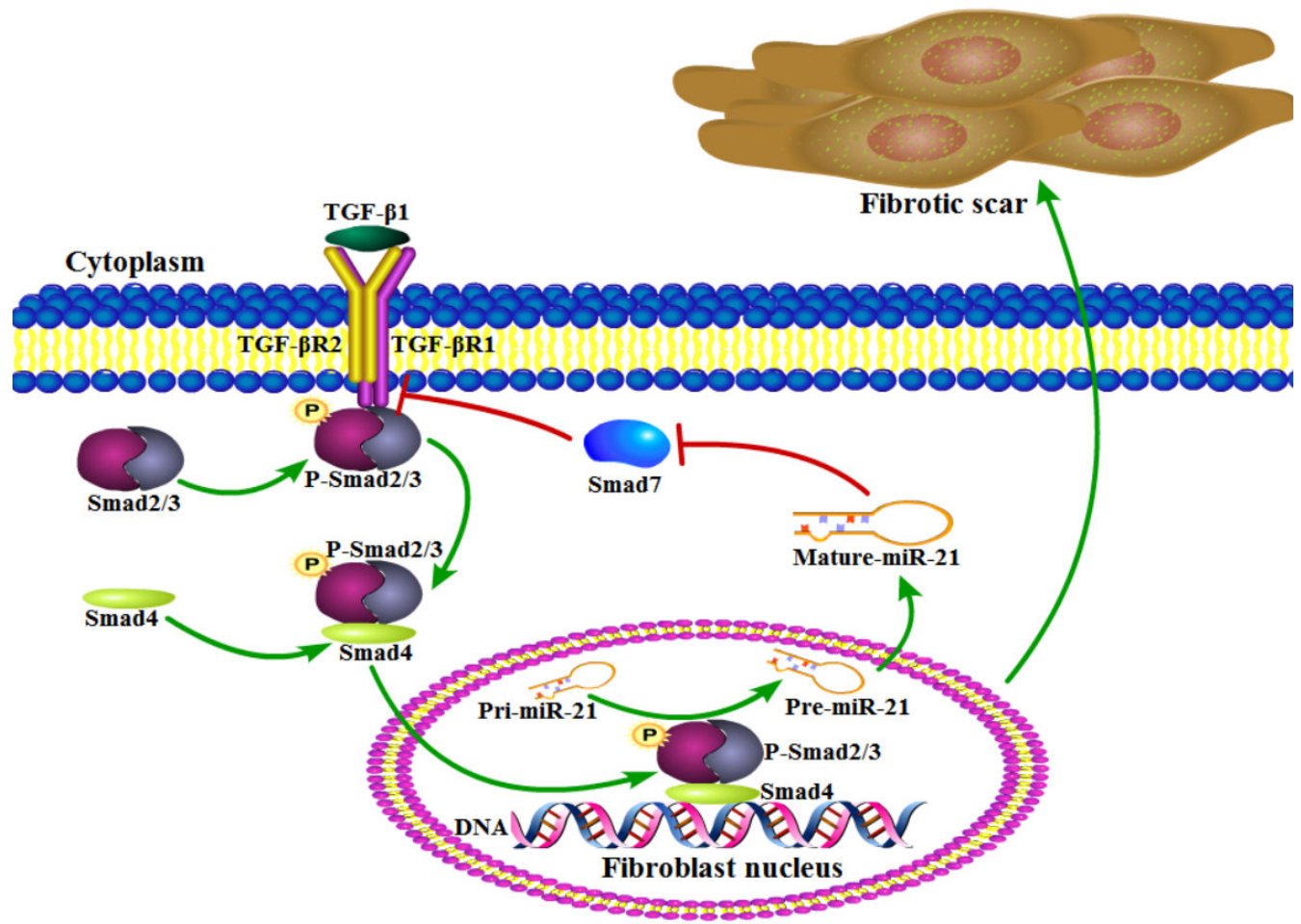

Figure 5. Working model of an amplifying circuit in the activation of spinal fibroblasts. TGF- $\beta$ complex phosphorylates Smad $2 / 3$, the phosphorylated Smad $2 / 3$ binding to Smad4 form a complex. Then the complex enters the nucleus forms a Drosha complex with DNA helicase, which promotes the conversion of pri-miR-21-5p into pre-miR-21-5p and accelerates miR-21-5p maturation. Smad7 which can be targeted and suppressed by miR-21-5p, protects Smad2/3 from being activated by TGF- $\beta$ R1. miR-21 was capable of enhancing TGF- $\beta$ signaling activation through an amplifying crosstalk.

Several studies have previously indicated that miR-21-5p is a pro-fibrotic miRNA expressed in many organs, including the kidney, lung, heart, and skin[19]. miRNAs normally have multiple targets, and miR-21-5p is known to contribute to fibrosis by repressing Sprouty1 and Phosphatase and Tensin Homolog deleted on chromosome 10[36]. Though Smad7 was previously shown to negatively regulate lung and skin fibrosis[11, 24], its role in fibrotic scar formation after SCI was unknown. Here, we demonstrated for the first time to our knowledge that Smad7 is a critical mediator of TGF- $\beta /$ miR-21 signaling during spinal fibroblast activation. Using cultured primary spinal fibroblasts, we showed that miR-21-5p regulates the collagen content by inhibiting Smad7 expression, thus contributing to fibrosis. It was previously reported that downstream effectors of TGF- $\beta$ signaling control the post-transcriptional processing of miR-21-5p through a direct protein interaction in the pre-miR-21 maturation complex. p-Smad2 and p-Smad3 promote the transcriptional activation of pri-miR-21, and regulate the subsequent post-transcriptional conversion of pri-miR-21 into pre-miR-21[37]. Here, we demonstrated that miR-21 was induced by TGF- $\beta$, which in turn promoted the TGF- $\beta$-regulated fibrotic activation of spinal fibroblasts by targeting Smad7 (Figure 5). miR-21 was capable of enhancing TGF- $\beta$ signaling activation through an amplifying circuit and promoting the formation of fibrotic scars after SCI; however, knockdown of miR-21 attenuated the pro-fibrotic activity of spinal fibroblasts in vivo.

We also investigated the roles of miR-21-5p in motor function recovery procedures after SCI, by knocking down miR-21-5p in mouse models and evaluating locomotor activity for 14 days post-surgery. Our results suggested that miR-21-5p knockdown significantly improved motor functional recovery after SCI. Furthermore, the expression of CSPGs, which inhibit neural regeneration, was suppressed in the miR-21-5p knockdown group, while the secretion of neural growth factors including NGF and BGNF was promoted. Although the amplifying circuit between miR-21-5p and the TGF- $\beta$ pathway is an important mediator of the fibrotic activation of spinal fibroblasts, it may not be the only factor causing poor prognosis after SCI. However, it remains unclear how miR-21-5p directly functions in spinal neuron cells and neuroglial cells. The data of KEGG have multiple implications and may open a series of opportunities. Interestingly, activin A receptor type IIB, a target gene of miR-21-5p and part of the TGF- $\beta$ pathway based on our KEGG data, may play a crucial role in axonal regeneration[27]. In conclusion, we demonstrated the molecular mechanism involved in the association of miR-21-5p 
and the TGF- $\beta$ / Smad pathway. We also showed the role of miR-21-5p in the formation of fibrotic scars and axonal regeneration after SCI by in vitro and in vivo studies. Our results have implications for therapeutic strategies in attenuating fibrotic scar formation and improving functional outcomes after SCI.

\section{Acknowledgements}

We are grateful to Dr. Yanan Zhao, Dr. Mingjie Sun, and Dr. Wenyue Cao for their valuable discussions and technical assistance. Grant support was provided by the National Natural Science Funds of China (Nos. 81401014, 81771346), the Chinese Postdoctoral Science Foundation (No. 2014M561935), and the Chinese Postdoctoral Science Foundation (No. 2015T80725).

\section{Authors' contributions}

$\mathrm{W}, \mathrm{W}$ performed the experiments. R,L participated in the design of the study and carried out the animal model. Y,S and H,L participated in the data analysis. B, $\mathrm{N}$ conceived of the study, and participated in its design and coordination and helped to draft the manuscript. All authors read and approved the final manuscript and they all declare that no competing interests exist.

\section{Competing Interests}

The authors have declared that no competing interest exists.

\section{References}

1. Alilain WJ, Horn KP, Hu H, Dick TE, Silver J. Functional regeneration of respiratory pathways after spinal cord injury. Nature. 2011; 475: 196-200.

2. Cristante AF, Barros Filho TE, Marcon RM, Letaif OB, Rocha ID. Therapeutic approaches for spinal cord injury. Clinics. 2012; 67: 1219-24.

3. Kawano H, Kimura-Kuroda J, Komuta Y, Yoshioka N, Li HP, Kawamura K, et al. Role of the lesion scar in the response to damage and repair of the central nervous system. Cell and tissue research. 2012; 349: 169-80.

4. Zhu Y, Soderblom C, Krishnan V, Ashbaugh J, Bethea JR, Lee JK. Hematogenous macrophage depletion reduces the fibrotic scar and increases axonal growth after spinal cord injury. Neurobiology of disease. 2015; 74: 114-25.

5. Soderblom C, Luo X, Blumenthal E, Bray E, Lyapichev K, Ramos J, et al. Perivascular fibroblasts form the fibrotic scar after contusive spinal cord injury. The Journal of neuroscience: the official journal of the Society for Neuroscience. 2013; 33: 13882-7.

6. Joko M, Osuka K, Usuda N, Atsuzawa K, Aoyama M, Takayasu M. Different modifications of phosphorylated Smad3C and Smad3L through TGF-beta after spinal cord iniury in mice. Neuroscience letters. 2013: 549: $168-72$

7. Butz H, Racz K, Hunyady L, Patocs A. Crosstalk between TGF-beta signaling and the microRNA machinery. Trends in pharmacological sciences. 2012; 33: $382-93$

8. Xu Q, Norman JT, Shrivastav S, Lucio-Cazana J, Kopp JB. In vitro models of TGF-beta-induced fibrosis suitable for high-throughput screening of antifibrotic agents. American journal of physiology Renal physiology. 2007; 293: F631-40.

9. Boche D, Cunningham C, Gauldie J, Perry VH. Transforming growth factor-beta 1-mediated neuroprotection against excitotoxic injury in vivo. Journal of cerebral blood flow and metabolism: official journal of the International Society of Cerebral Blood Flow and Metabolism. 2003; 23: $1174-82$.

10. Makwana M, Jones LL, Cuthill D, Heuer H, Bohatschek M, Hristova M, et al. Endogenous transforming growth factor beta 1 suppresses inflammation and promotes survival in adult CNS. The Journal of neuroscience: the official journal of the Society for Neuroscience. 2007; 27: 11201-13.
11. Zhu $\mathrm{H}$, Luo $\mathrm{H}$, Li $\mathrm{Y}$, Zhou $\mathrm{Y}$, Jiang $\mathrm{Y}$, Chai J, et al. MicroRNA-21 in scleroderma fibrosis and its function in TGF-beta-regulated fibrosis-related genes expression. Journal of clinical immunology. 2013; 33: 1100-9.

12. Laudisi F, Dinallo V, Di Fusco D, Monteleone G. Smad7 and its Potential as Therapeutic Target in Inflammatory Bowel Diseases. Current drug metabolism. 2016; 17: 303-6.

13. Dong H, Lei J, Ding L, Wen Y, Ju H, Zhang X. MicroRNA: function, detection, and bioanalysis. Chemical reviews. 2013; 113: 6207-33.

14. Bhalala OG, Srikanth M, Kessler JA. The emerging roles of microRNAs in CNS injuries. Nature reviews Neurology. 2013; 9: 328-39.

15. Cloutier F, Marrero A, O'Connell C, Morin P, Jr. MicroRNAs as potential circulating biomarkers for amyotrophic lateral sclerosis. Journal of molecular neuroscience: MN. 2015; 56: 102-12.

16. Ameres SL, Zamore PD. Diversifying microRNA sequence and function. Nature reviews Molecular cell biology. 2013; 14: 475-88.

17. Stoicea N, Du A, Lakis DC, Tipton C, Arias-Morales CE, Bergese SD. The MiRNA Journey from Theory to Practice as a CNS Biomarker. Frontiers in genetics. 2016; 7: 11

18. Huang $\mathrm{Y}, \mathrm{He} \mathrm{Y}, \mathrm{Li}$ J. MicroRNA-21: a central regulator of fibrotic diseases via various targets. Current pharmaceutical design. 2015; 21: 2236-42.

19. Liu RH, Ning B, Ma XE, Gong WM, Jia TH. Regulatory roles of microRNA-21 in fibrosis through interaction with diverse pathways (Review). Molecular medicine reports. 2016; 13: 2359-66.

20. Ning B, Gao L, Liu RH, Liu Y, Zhang NS, Chen ZY. microRNAs in spinal cord injury: potential roles and therapeutic implications. International journal of biological sciences. 2014; 10: 997-1006.

21. Bhalala OG, Pan L, Sahni V, McGuire TL, Gruner K, Tourtellotte WG, et al. microRNA-21 regulates astrocytic response following spinal cord injury. The Journal of neuroscience: the official journal of the Society for Neuroscience. 2012; 32: 17935-47.

22. Krutzfeldt J, Rajewsky N, Braich R, Rajeev KG, Tuschl T, Manoharan M, et al. Silencing of microRNAs in vivo with 'antagomirs'. Nature. 2005; 438: 685-9.

23. Komuta Y, Teng X, Yanagisawa H, Sango K, Kawamura K, Kawano H. Expression of transforming growth factor-beta receptors in meningeal fibroblasts of the injured mouse brain. Cellular and molecular neurobiology. 2010; 30: 101-11.

24. Liu G, Friggeri A, Yang Y, Milosevic J, Ding Q, Thannickal VJ, et al. miR-21 mediates fibrogenic activation of pulmonary fibroblasts and lung fibrosis. The Journal of experimental medicine. 2010; 207: 1589-97.

25. Yiu G, He Z. Glial inhibition of CNS axon regeneration. Nature reviews Neuroscience. 2006; 7: 617-27.

26. Sun F, Park KK, Belin S, Wang D, Lu T, Chen G, et al. Sustained axon regeneration induced by co-deletion of PTEN and SOCS3. Nature. 2011; 480: 372-5.

27. Omura T, Omura K, Tedeschi A, Riva P, Painter MW, Rojas L, et al. Robust Axonal Regeneration Occurs in the Injured CAST/Ei Mouse CNS. Neuron. 2015; 86: 1215-27.

28. Silver J, Miller JH. Regeneration beyond the glial scar. Nature reviews Neuroscience. 2004; 5: 146-56.

29. Anderson MA, Burda JE, Ren Y, Ao Y, O'Shea TM, Kawaguchi R, et al. Astrocyte scar formation aids central nervous system axon regeneration. Nature. 2016; 532: 195-200.

30. Shechter R, Raposo C, London A, Sagi I, Schwartz M. The glial scar-monocyte interplay: a pivotal resolution phase in spinal cord repair. PloS one. 2011; 6: e27969.

31. Liddelow SA, Barres BA. Regeneration: Not everything is scary about a glial scar. Nature. 2016; 532: 182-3.

32. Klapka N, Muller HW. Collagen matrix in spinal cord injury. Journal of neurotrauma. 2006; 23: 422-35.

33. Hara M, Kobayakawa K, Ohkawa Y, Kumamaru H, Yokota K, Saito T, et al. Interaction of reactive astrocytes with type I collagen induces astrocytic scar formation through the integrin-N-cadherin pathway after spinal cord injury. Nature medicine. 2017; 23: 818-28.

34. Jiang Y, Wang C, Li YY, Wang XC, An JD, Wang YJ, et al. Mistletoe alkaloid fractions alleviates carbon tetrachloride-induced liver fibrosis through inhibition of hepatic stellate cell activation via TGF-beta/Smad interference. Journal of ethnopharmacology. 2014; 158 (Pt A): 230-8.

35. Li Q, Zhang D, Wang Y, Sun P, Hou X, Larner J, et al. MiR-21/Smad 7 signaling determines TGF-beta1-induced CAF formation. Scientific reports. 2013; 3: 2038.

36. Liang H, Zhang C, Ban T, Liu Y, Mei L, Piao X, et al. A novel reciprocal loop between microRNA-21 and TGFbetaRIII is involved in cardiac fibrosis. The international journal of biochemistry \& cell biology. 2012; 44: 2152-60.

37. Garcia R, Nistal JF, Merino D, Price NL, Fernandez-Hernando C, Beaumont J, et al. p-SMAD2/3 and DICER promote pre-miR-21 processing during pressure overload-associated myocardial remodeling. Biochimica et biophysica acta. 2015; 1852: 1520-30. 